\title{
Low prevalence of human papillomavirus in squamous-cell carcinoma limited to oral cavity proper
}

\author{
Luca Scapoli ${ }^{1}$, Annalisa Palmieri ${ }^{1}$, Corrado Rubini ${ }^{2}$, Marcella Martinelli ${ }^{1}$, \\ Giuseppe Spinelli ${ }^{3}$, Franco Ionna ${ }^{4}$ and Francesco Carinci ${ }^{5}$
}

${ }^{1}$ Department of Histology, Embryology and Applied Biology, Centre of Molecular Genetics, University of Bologna, Bologna, Italy; ${ }^{2}$ Anatomical Pathology Unit, Polytechnic University of the Marche Region, Ancona, Italy; ${ }^{3}$ Section of Maxillo-Facial Surgery, Careggi Hospital, Firenze, Italy; ${ }^{4}$ Maxillo-Facial and ENT Department, 'G. Pascale’ National Institute of Tumours, Naples, Italy and ${ }^{5}$ Department of D.M.C.C.C., Section of Maxillo-Facial Surgery, University of Ferrara, Ferrara, Italy

\begin{abstract}
Several investigations have reported that the human papillomavirus may play a role in head and neck squamous-cell carcinoma development and may have a prognostic impact. However, inconsistent results regarding human papillomavirus prevalence have been obtained so far. Variables which may account for these discrepancies may be related to site of the samples' origin, detection methods and sample size. The aim of this study is to evaluate the presence of high-risk type human papillomavirus in a large, well defined sample of squamous-cell carcinoma limited to the oral cavity in its strictest definition-ie with no pharynx or larynx cancers included-by means of quantitative real-time polymerase chain reaction, a method which ensures high sensitivity and offers the opportunity to exclude false-positive results. Data were obtained from 314 squamouscell carcinoma limited to oral cavity proper, indicated that the prevalence of high-risk human papillomavirus was as low as $2 \%(\mathrm{Cl} 0.6-3)$. Matched pairs case-control analysis indicated that the prevalence among controls did not significantly differ with respect to cases and thus did not support a major role for the human papillomavirus in the etiology of squamous-cell carcinoma of the oral cavity.
\end{abstract}

Modern Pathology (2009) 22, 366-372; doi:10.1038/modpathol.2008.180; published online 31 October 2008

Keywords: squamous-cell carcinoma; oral cavity; quantitative real-time polymerase chain reaction; human papillomavirus

Head and neck squamous-cell carcinoma is a major health problem worldwide, accounting for over 300000 incident cases annually. ${ }^{1}$ Among head and neck oncological sites (ie oral cavity, pharynx, larynx and paranasal sinuses), squamous-cell carcinoma limited to the oral cavity in its strictest definition (ie behind the tonsils) accounts for the vast majority of cases. ${ }^{1}$

The behavior of squamous-cell carcinoma depends on several variables. ${ }^{2}$ Metastases, which occur via the lymphatics, are the main cause of death; their incidence is significantly correlated to the localization and the clinical stages of the

Correspondence: Dr L Scapoli, PhD, Department of Histology, Embryology and Applied Biology, University of Bologna, Via Belmeloro 8, Bologna 40126, Italy.

E-mail: luca.scapoli2@unibo.it

Received 4 September 2008; revised 26 September 2008; accepted 29 September 2008; published online 31 October 2008 primary tumor, as well as the degree of deep infiltration and cell differentiation. ${ }^{3-5}$

Tobacco and alcohol consumption have been identified as the most important determinants for the development of squamous-cell carcinoma. ${ }^{6,7}$ The human papillomavirus infection has also been postulated as a risk factor for squamous-cell carcinoma, although conflicting results have been reported. Indeed, substantial molecular evidence supports the role of the human papillomavirus in the pathogenesis of oropharyngeal squamous-cell carcinoma, especially regarding tonsil carcinomas, with average prevalence rates ranging from 12 to $70 \%,{ }^{8-11}$ whereas squamous-cell carcinoma of the oral cavity itself has a lower prevalence rate, ranging between 3 and $15 \%{ }^{10,12,13}$ The wide range of prevalence could be related to (1) the distribution of the human papillomavirus among the population, (2) oral sexual behavior, (3) the sampling strategy used (gross specimens, cotton swaps or mouthwash) $)^{14-17}$ and (4) the detection method 
(polymerase chain reaction, quantitative polymerase chain reaction, enzyme-linked immunosorbent assay and immunohistochemistry). ${ }^{18,19}$ In addition, random fluctuations due to limited-sized sampling have exacerbated variability. ${ }^{20}$

To detect whether the human papillomavirus might have a role in the onset of squamous-cell carcinoma limited to oral cavity proper, a large series of fresh and paraffin-embedded tumor specimens with pair-matched controls were analyzed by means of quantitative polymerase chain reactions and the results verified with immunohistochemistry.

\section{Materials and methods}

\section{Tissue Collection}

Patients were diagnosed between 1990 and 2007, at the Anatomical Pathology Unit, Polytechnic University of the Marche Region, Ancona, Italy. The sample was composed of 280 squamous-cell carcinoma limited to the oral cavity in its strictest definition-ie no tonsil, pharynx or larynx cancers were included-and matched control pairs. Of them, 237 matched pairs were obtained from paraffin-embedded specimens, whereas 43 were freshly frozen. In addition, 57 paraffin-embedded tumors without matched controls were analyzed. The clinical features of the enrolled cases have been summarized in Figures 1 and 2. Tumor samples included at least $80 \%$ neoplastic cells and control tissues were obtained from the same patient and anatomical region.

\section{DNA Extraction}

DNA from paraffin-embedded samples was extracted from two $12 \mu \mathrm{m}$ thick sections. Slices were placed in xylol for $1 \mathrm{~h}$ and centrifuged at $12000 \mathrm{~g}$. DNA extraction and purification was performed using the NucleoSpin Tissue Kit (Macherey-Nagel), which can recover DNA fragments as short as $200 \mathrm{bp}$. The tissue was washed with absolute ethanol twice then incubated overnight at $56^{\circ} \mathrm{C}$ in $200 \mu \mathrm{l}$ of lysis solution with $1 \mathrm{mg} / \mathrm{ml}$ of proteinase $\mathrm{K}$ until it was completely dissolved. Proteinase $\mathrm{K}$ was inactivated by heating it to $70^{\circ} \mathrm{C}$ for $10 \mathrm{~min}$. Cellular debris was removed by spinning at $12000 \mathrm{~g}$ for 5 min. The DNA was then purified with NucleoSpin Tissue columns. Fresh tissue was treated in as

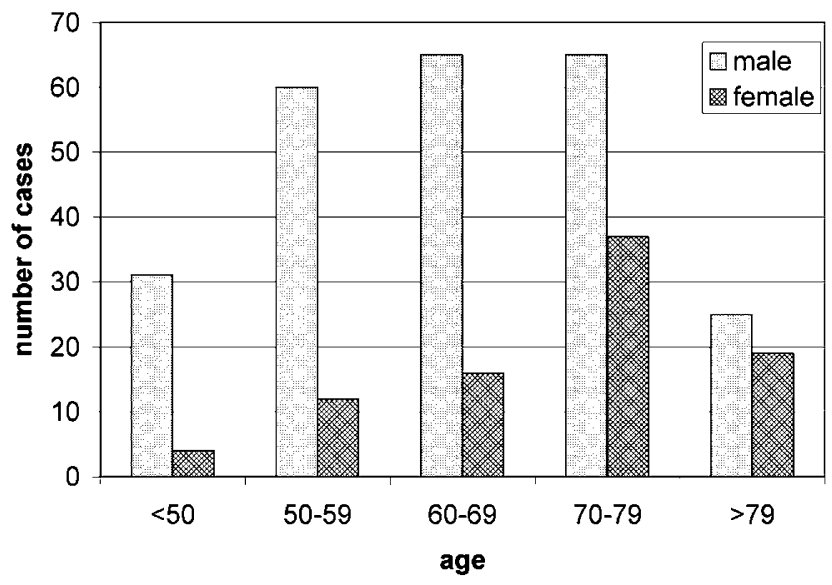

Figure 1 Sample cases by age and sex.

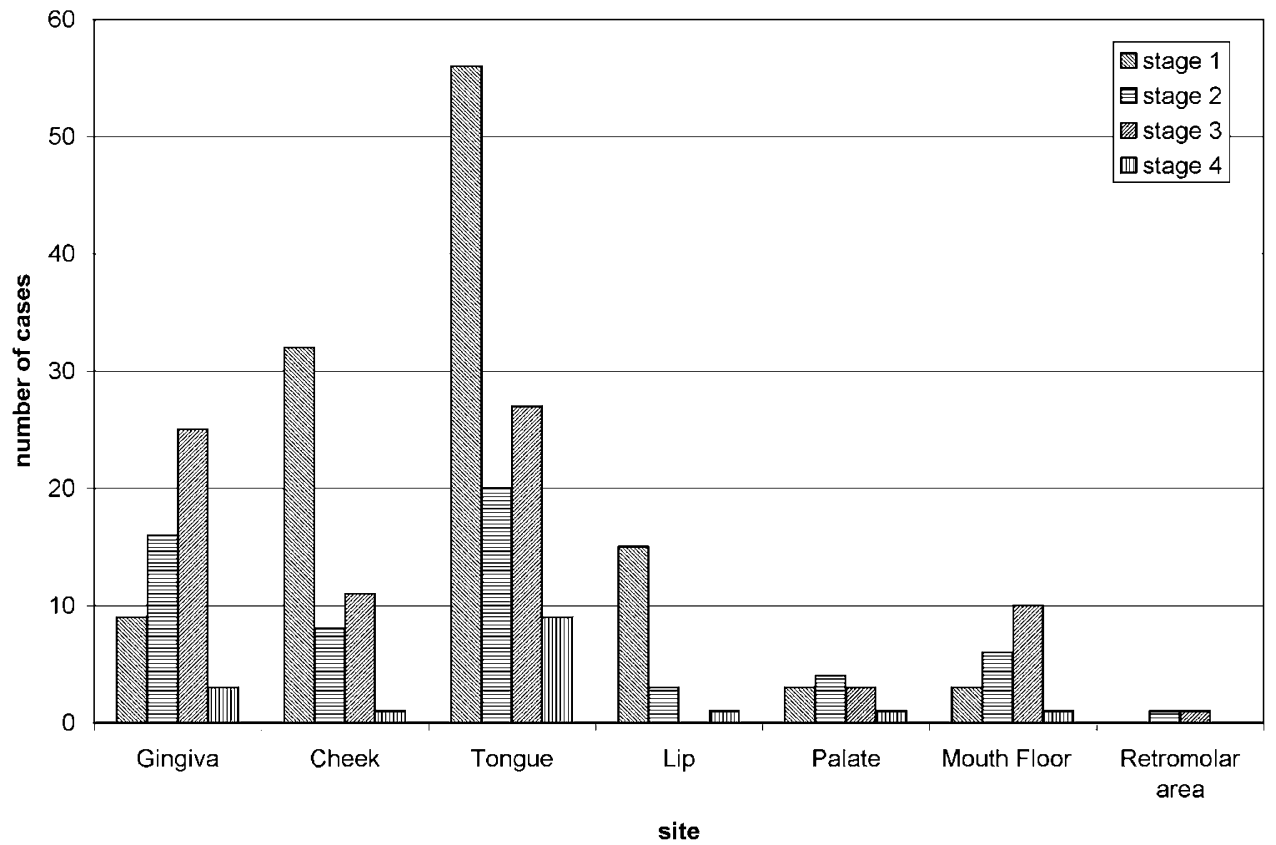

Figure 2 Clinical features of enrolled cases. 
described, although these samples did not require xylene treatment and ethanol washing.

\section{Primers and Probes}

The primer and probe sequences used to simultaneously detect human papillomavirus types 16, 18, 31 and 45 in a single tube reaction, were those previously described by Moberg et al, ${ }^{21}$ although a different combination of dyes and quenchers was adopted to account for the different real-time polymerase chain reaction apparatus. TaqMan probes for the detection of human papillomavirus 16 and -31 were quenched with the nonfluorescent dye Black Hole Quencer 1 (BHQ1) at the $3^{\prime}$ end, and labeled at the $5^{\prime}$ end with the fluorescent dyes FAM and JOE, respectively. Human papillomavirus 18 and -45 were detected and quantified together with a single probe quenched with the Black Berry Quencher (BBQ-650) and labeled with Cyanine 5 (Biomers, Ulm, Germany).

The primers and probe for the amplification of a unique sequence in the first intron of the human gene hydroxymethylbilane synthase isoform 2 (HMBS) were designed using the Primer Express software (Applied Biosystems) (HMBSf: 5'-AAGA CACGTTCCACTTTTGATTCA-3'; HMBSr: 5'-ACA CAAAAAGAAGGCGCACTTC- $3^{\prime}$; HMBSProbe: 5'AAGCCTCCGAACTGCACACAAACGTC- $3^{\prime}$ ). The probe was quenched with BHQ1 at the $3^{\prime}$ end and labeled with JOE at the $5^{\prime}$ end.

\section{Real-Time Polymerase Chain Reaction}

Two real-time polymerase chain reaction runs were performed for each sample. The first reaction detected and quantified the amount of the human single copy gene HMBS and included two primers and a single probe. The second reaction detected and quantified human papillomavirus $16,-31$ and -18 together with -45 . This reaction included a total of seven polymerase chain reaction primers and three probes. ${ }^{21}$ Absolute quantification assays were performed using the Applied Biosystems 7500 Sequence Detection System. Each reaction was performed in $20 \mu \mathrm{l}$ containing $10 \mu \mathrm{l}$ of $2 \times$ TaqMan universal polymerase chain reaction master mix (Applied Biosystems), $4 \mu \mathrm{l}$ of DNA purified from samples and $200 \mathrm{nM}$ of each primer and probe, with the exception of the human papillomavirus 16 probe used at $100 \mathrm{nM}$. The amplification profile was initiated by a $10 \mathrm{~min}$ incubation period at $95^{\circ} \mathrm{C}$, followed by a two-step amplification of $15 \mathrm{~s}$ at $95^{\circ} \mathrm{C}$ and $60 \mathrm{~s}$ at $57^{\circ} \mathrm{C}$ for 40 cycles. All these experiments were performed including nontemplate controls to exclude reagents contamination. The instruments worked in '9600 emulation' modality to mimic the characteristic of the ABI PRISM 7700 Sequence Detection System used to set the assay. ${ }^{21}$
Standard curves for the human papillomavirus types (16, 31 and 45/18) were constructed in a triplex reaction, by using a mix of the same amount of human papillomavirus $16,-18$ and -31 plasmids, in serial dilutions ranging from $10^{1}$ to $10^{7}$ copies. There was a linear relationship between the threshold cycle values plotted against the log of the copy number over the entire range of dilutions (data not shown). Plasmids containing human papillomavirus $16,-18$ and -45 DNA were kindly supplied by EM de Viliers (German Cancer Research Centre), the plasmid containing human papillomavirus -31 DNA was kindly supplied by A Lorincz (Digene Corporation). The plasmids' DNA was transfected in competent bacteria and extracted using the Qiagen Plasmid Mini Kit (QIAGEN). The copy numbers for individual plasmid preparations were estimated using the Quant.iT dsDNA BR assay kit (Invitrogen) and the Qubit ${ }^{\mathrm{TM}}$ fluorometer (Invitrogen).

Standard curves for the human genome were created using serial dilutions with amounts ranging from $10^{1}$ to $10^{5}$ of the genomic DNA copies, which had been extracted from blood using the GenElute Blood Genomic DNA Kit (Sigma-Aldrich) and quantified as described above. The absolute quantification of genome copies in samples allowed for the quantification of the input cell number and the calculation of the viral load, which is the number of viral genomes per cell. Samples were scored as positive for human papillomavirus when the viral load was $>0.1$ ( $>1$ human papillomavirus genome copy/10 cells) as explained previously. ${ }^{12}$ This threshold was based on the assumption that the presence of human papillomavirus at a level below the threshold indicated that there was not a sufficient amount of human papillomavirus to play a major role in the earliest stages of tumor initiation and progression.

To prevent samples and polymerase chain reaction contamination, plasmid purification and handling was performed in a separate laboratory with dedicated pipettes.

\section{Statistic Analyses}

Descriptive statistics was performed using Excel spreadsheets (Microsoft Office 2003). Exact Mid-P testing for proportion and for matched pair casecontrol analysis was performed online at the OpenEpi web site (www.openepi.com).

\section{In Situ Hybridization}

In situ hybridization was performed with three separate probes for the human papillomavirus, using the DakoCytomation GenPoint Tyramide Signal Amplification System (DakoCytomation, Carpinteria, CA, USA). A paraffin-embedded section of a low-grade squamous intraepithelial lesion confirmed as positive for high-risk human papillo- 
mavirus was included as a positive external control in each run. Nondysplastic squamous mucosa was included as a negative external control.

The in situ hybridization assay was performed on $4 \mu \mathrm{m}$ thick sections using an antigen retrieval citrate buffer system at a dilution of 1:500. Slides were incubated in proteinase $\mathrm{K}$ solution for $5 \mathrm{~min}$ at room temperature, washed with water, then quenched with $0.3 \% \mathrm{H}_{2} \mathrm{O}_{2}$ in methanol and washed again. Excess liquid was removed by air drying and 1 drop $(15 \mu \mathrm{l})$ of the human papillomavirus probe solution was applied. Cover-slipped slides were denaturated on a hot plate at $90^{\circ} \mathrm{C}$ for $8 \mathrm{~min}$, and then incubated in a $37^{\circ} \mathrm{C}$ oven overnight. Slides were soaked in Trisbuffered saline Tween 20 for 5 min, treated with Stringent Wash solution (diluted $1: 50$ ) at $48^{\circ} \mathrm{C}$ for $30 \mathrm{~min}$ and washed several times with Tris-buffered saline Tween 20. Detection was performed using the DakoCytomation GenPoint Tyramide Signal Amplification System. Counterstaining was performed with Mayer's hematoxylin.

DakoCytomation nuclear staining was evaluated as either positive or negative using two pathologists. In addition, positive nuclear staining was further characterized as punctuated, diffused or mixed.

\section{Results}

The specificity and sensitivity of the quantitative polymerase chain reaction assay developed by Moberg et $a l^{21}$ as well as its ability to detect mixed infection for four types of human papillomavirus $(-16,-31,-45$ and -18$)$ was confirmed in our laboratory with a series of preliminary experiments. Small amounts of human papillomavirus plasmids were added to 150 randomly selected squamous-cell carcinoma DNA samples to test for the absence of contaminants that may have inhibited polymerase chain reaction. The sensitivity of the assay was not significantly altered with respect to the same amount of DNA in pure water.

A fixed volume of purified DNA from each sample was tested in two quantitative real-time polymerase chain reactions to evaluate the number of cells and the number of human papillomavirus genomes in each reaction. The cell number was evaluated via the quantification of a single copy genomic sequence, which is located in the HMBS locus. Reactions involving less than 100 cells were excluded from the analysis because these could produce false negative results due to low viral loads. Of 337 tumor tissues, 23 and 25 of 280 controls resulted as below the threshold. All of these were from the paraffin-embedded specimens. Among the 569 remaining samples, the median analyzed cell number in each reaction was 1941 (interquartile range 602-7295).

Of 569 samples, 7, including tumors and controls, were found to be human papillomavirus 16 positive (Table 1), whereas none resulted positive for human
Table 1 Description of cases and control matched pairs, positive for human papillomavirus 16

\begin{tabular}{lclcc}
\hline$T$ & N & Site & Case viral load & Matched control viral load \\
\hline 2 & + & Floor & 102 & Negative \\
1 & 0 & Cheek & 0.18 & Negative $(<0.1)$ \\
1 & 0 & Cheek & 0.417 & Not available \\
1 & + & Cheek & 4.115 & 19.07 \\
2 & + & Tongue & 28.118 & Negative $(<0.1)$ \\
Normal & - & Tongue & Negative & 0.33 \\
\hline
\end{tabular}

Table 2 Pair-matched case-control study for the presence of human papillomavirus 16 in squamous cell carcinoma

\begin{tabular}{|c|c|c|c|}
\hline & \multicolumn{2}{|c|}{ Controls } & \multirow[t]{2}{*}{ Total } \\
\hline & $(+)$ & $(-)$ & \\
\hline \multicolumn{4}{|l|}{ Cases } \\
\hline$(+)$ & 1 & 3 & 4 \\
\hline$(-)$ & 1 & 230 & 231 \\
\hline Total & 2 & 233 & 235 \\
\hline
\end{tabular}

papillomavirus $18,-31$ or -45 . Human papillomavirus 16 was detected in 5 tumors out of the 314 examined. The prevalence rate for human papillomavirus 16 was $2 \%$ (CI $0.6-3$ ). The matched casecontrols analysis $(n=235$; Table 2) demonstrated that the human papillomavirus did not correlate with squamous-cell carcinoma $(P$-value $=0.37)$; the conditional maximum likelihood estimate of odds ratio $=3$ (CI 0.3-79). Among unmatched cases and controls, 1 of 79 squamous-cell carcinoma and none of the 20 controls were human papillomavirus positive $(P$-value $=0.40)$. Interestingly, human papillomavirus 16 was not detected in any of the 43 matched pairs, which provided high quality DNA as it was extracted from frozen tissue.

Of the samples, 35 (13 tumors and 22 controls) showed some endpoint signals, but were scored as negative for the human papillomavirus as they did not reach the threshold of 0.1 viral genomes per cell. For the majority of these samples the viral load was very low, in the order of $10^{-3}-10^{-4}$ viral genomes per cell.

The five tumors found to be human papillomavirus positive using real-time polymerase chain reaction were reviewed by pathologists and further investigated using DNA in situ hybridization. No koilocytotic lesions or lymphatic tissues were observed in these samples. The sample with the highest viral load ( $>100$ viral genome/cell) demonstrated punctuated human papillomavirus positivity by in situ hybridization, which was specifically localized in the nucleus of the tumor cells (Figure 3).

\section{Discussion}

Human papillomavirus is associated with a variety of benign and malignant skin and mucosa tumors. 


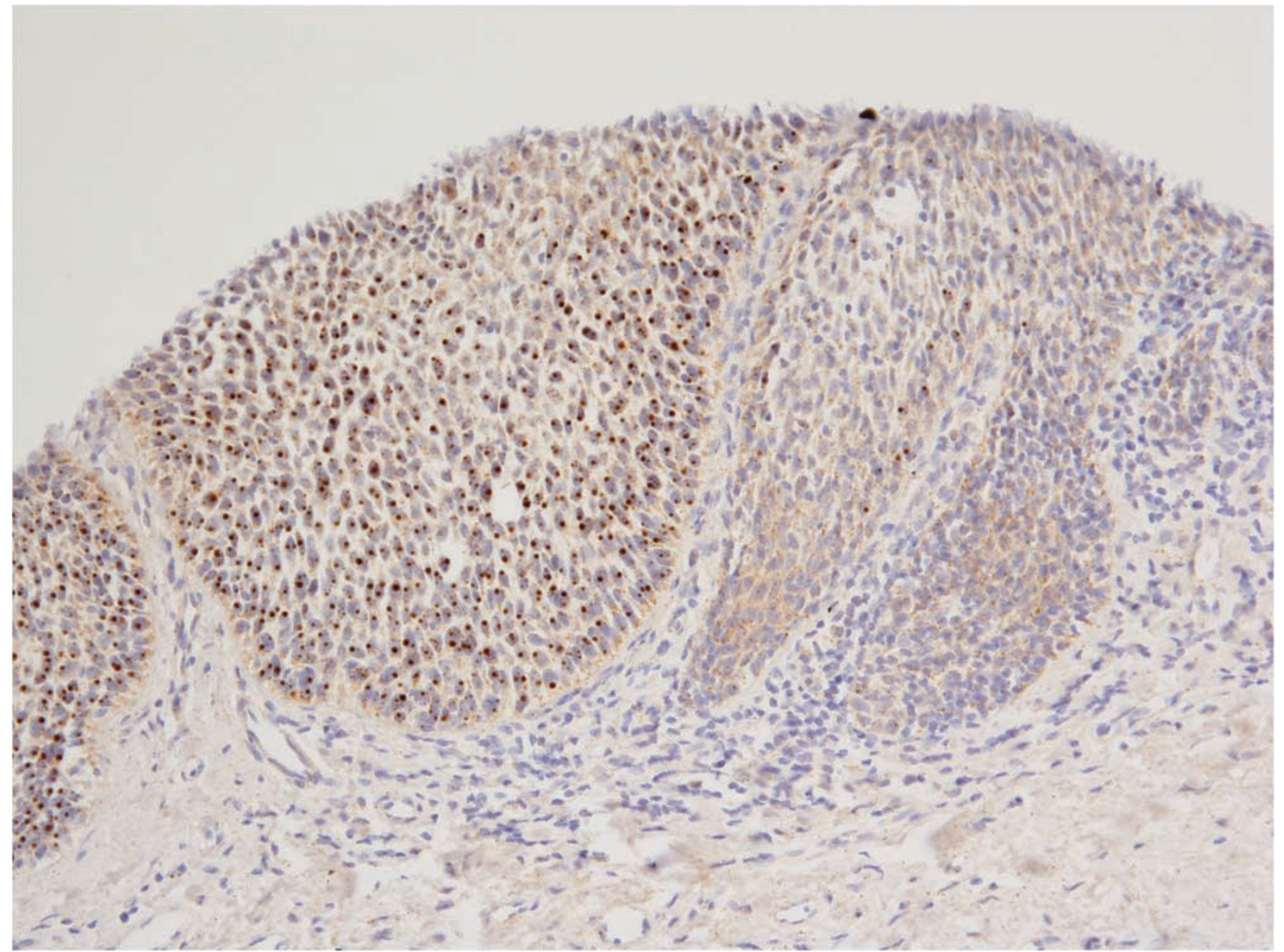

Figure 3 Detection of high-risk human papillomavirus with DakoCytomation. Punctuated positive nuclear staining in squamous-cell carcinoma.

High-risk viral types of human papillomavirus are the major cause of cervical carcinoma but are frequently found in carcinomas from different areas, including the head and neck. In this study, we tested for the presence of four high-risk human papillomavirus $(-16,-45,-18$ and -31$)$ in a large sample of squamous-cell carcinomas limited to oral cavity proper and matched controls, using quantitative real-time polymerase chain reaction.

Among the 569 samples (314 cases and 255 controls), 7 demonstrated clinically significant amounts of human papillomavirus -16 DNA, whereas human papillomavirus $18,-31$ and -45 were not detected at all. Human papillomavirus 16 prevalence in squamous-cell carcinoma strictly limited to the oral cavity was as low as $2 \%$; moreover, there was no significantly difference with respect to normal tissue located near the tumor. The presence of human papillomavirus 16 was confirmed by in situ hybridization for a sample with a high viral load ( $>100$ viral genomes per cell), whereas no hybridization signal was observed in samples with a lower viral load. These data may be due to the differences in the sensitivity of the two techniques, but may also depend on other factors. For instance, it is known that in situ hybridization sensitivity for human papillomavirus detection decreases if biopsies are not processed quickly. Samples used in this investigation had been collected specifically for this study, not for in situ hybridization experiments, so no particular care had been taken as regards this aspect. Overall, the data suggest that human papillomavirus does not play a significant role in the etiology of squamous-cell carcinoma limited to the oral cavity in its strictest definition, although we cannot exclude its contribution to carcinogenesis in a minority of cases.

A systematic review of the literature on the human papillomavirus in head and neck squamous-cell carcinoma has revealed a widespread prevalence variability that can only be in part due to the cancer site, geographic location and study sample size. ${ }^{20}$ A dissection of the data, indicated that the lowest prevalence was observed among oral cavity squamous-cell carcinoma in European samples and that larger studies tended to show a lower than the average overall human papillomavirus prevalence. ${ }^{20}$ The conclusions of our study are in 
line with the major studies which have shown a discrimination of human papillomavirus prevalence based on the tumor site of origin. Herrero et al reported that the prevalence of oral cavity squamous-cell carcinoma was $3.9 \%$ in 766 cases, whereas Dahlgren et al obtained a value of $2.3 \% .^{10,22}$

It is known that human papillomavirus prevalence varies among different populations. It has been reported that the proportion of women infected with human papillomavirus ranges from $1.4 \%$ in Spain to $25.6 \%$ in Nigeria, whereas in Italy has an intermediate value of $9.2 \%{ }^{23}$ As human papillomavirus is widespread in the Italian population, we may argue that the low prevalence of human papillomavirus observed in this study among squamous-cell carcinoma as well as controls, may be due to its negligible contribution to carcinogenesis or to the low tropism of the virus for oral mucosa. On the other hand, the prevalence observed in this study was lower than that previously observed in other Italian investigations. It was, for instance, about $18 \%$ in samples derived from the saliva of healthy subjects, ${ }^{24} 18 \%$ in premalignant lesions ${ }^{25}$ and ranged from 13 to $36 \%$ in oral squamous-cell carcinomas (detected with small samples of approximately 50 cases). ${ }^{26,27}$

It has emerged that studies based on polymerase chain reaction assays have reported a higher prevalence than other less sensitive techniques such as Southern blot or in situ hybridization. ${ }^{15}$ One of the difficulties with polymerase chain reaction is its extreme sensitivity to contamination. However, this may simply reflect inherent methodological drawbacks. Even careful tissue extraction and handling does not necessarily prevent contamination; it may be virtually impossible to eliminate or account for using conventional techniques. An overestimation of human papillomavirus positivity due to technical limitations may also help explain conflicting reports. A real-time quantitative polymerase chain reaction assay was adopted during this investigation. This method is extremely sensitive but does allow for the differentiation of target contamination. We adopted a threshold of 0.1 human papillomavirus DNA copy per cell genome (1 copy/10 genome equivalents) which is the same value as used by $\mathrm{Ha}$ et $a l,{ }^{12}$ with the assumption that without sufficient copies of human papillomavirus affecting the majority of cells within a lesion, it is unlikely that human papillomavirus plays a major role in the carcinogenesis process. It is also possible that DNA extraction from paraffin-embedded tissue may decrease the likelihood of detection. To account for the differences in the quality of the DNA used, an internal control and low-quality samples were excluded. Furthermore, human papillomavirus was not detected in the 44 matched pair samples from fresh frozen tissue, implying that the tissue processing technique did not affect the results.

In previous studies on the Italian population, a high prevalence of human papillomavirus was detected in premalignant lesions. ${ }^{25}$ Our series is exclusively composed of squamous-cell carcinoma. Although certainly not ruling out the possible role of human papillomavirus in oral squamous-cell carcinoma, especially in the context of a 'hit and run' mechanism, it is noteworthy that in the oropharyngeal region, where human papillomavirus has a high prevalence, the virus is also detectable in advanced tumors. Consequently, our results suggest that human papillomavirus 16 infection in oral cavity carcinomas is not a frequent event and support the view that human papillomavirus is probably unimportant in the pathogenesis of squamous-cell carcinoma of the oral cavity proper.

\section{Acknowledgement}

This work was supported by Associazione Tumori Toscana, Fondazione Cassa di Risparmio di Ferrara and the Italian Ministry of Universities and Scientific Research.

\section{References}

1 Parkin DM, Pisani P, Ferlay J. Estimates of the worldwide incidence of 25 major cancers in 1990. Int J Cancer 1999;80:827-841.

2 Carinci F, Pelucchi S, Farina A, et al. Site-dependent survival in cancer of the oral cavity. J Craniofac Surg 1997;8:399-403; discussion 404.

3 O'Brien CJ, Lauer CS, Fredricks S, et al. Tumor thickness influences prognosis of $\mathrm{T} 1$ and $\mathrm{T} 2$ oral cavity cancer-but what thickness? Head Neck 2003;25:937-945.

4 O-charoenrat P, Pillai G, Patel S, et al. Tumour thickness predicts cervical nodal metastases and survival in early oral tongue cancer. Oral Oncol 2003;39:386-390.

5 Po Wing Yuen A, Lam KY, Lam LK, et al. Prognostic factors of clinically stage I and II oral tongue carcinoma-A comparative study of stage, thickness, shape, growth pattern, invasive front malignancy grading, Martinez-Gimeno score, and pathologic features. Head Neck 2002;24:513-520.

6 Blot WJ, McLaughlin JK, Winn DM, et al. Smoking and drinking in relation to oral and pharyngeal cancer. Cancer Res 1988;48:3282-3287.

7 Schlecht NF, Franco EL, Pintos J, et al. Interaction between tobacco and alcohol consumption and the risk of cancers of the upper aero-digestive tract in Brazil. Am J Epidemiol 1999;150:1129-1137.

8 Charfi L, Jouffroy T, de Cremoux P, et al. Two types of squamous cell carcinoma of the palatine tonsil characterized by distinct etiology, molecular features and outcome. Cancer Lett 2008;260:72-78.

9 Chien CY, Su CY, Fang FM, et al. Lower prevalence but favorable survival for human papillomavirus-related squamous cell carcinoma of tonsil in Taiwan. Oral Oncol 2008;44:174-179.

10 Herrero R, Castellsague X, Pawlita M, et al. Human papillomavirus and oral cancer: the International 
Agency for Research on Cancer multicenter study. J Natl Cancer Inst 2003;95:1772-1783.

11 Kim SH, Koo BS, Kang S, et al. HPV integration begins in the tonsillar crypt and leads to the alteration of $\mathrm{p} 16$, EGFR and c-myc during tumor formation. Int J Cancer 2007;120:1418-1425.

12 Ha PK, Pai SI, Westra WH, et al. Real-time quantitative PCR demonstrates low prevalence of human papillomavirus type 16 in premalignant and malignant lesions of the oral cavity. Clin Cancer Res 2002;8: 1203-1209.

13 Nagpal JK, Patnaik S, Das BR. Prevalence of high-risk human papilloma virus types and its association with p53 codon 72 polymorphism in tobacco addicted oral squamous cell carcinoma (OSCC) patients of eastern India. Int J Cancer 2002;97:649-653.

14 Giovannelli L, Campisi G, Colella G, et al. Brushing of oral mucosa for diagnosis of HPV infection in patients with potentially malignant and malignant oral lesions. Mol Diagn Ther 2006;10:49-55.

15 Hansson BG, Rosenquist K, Antonsson A, et al. Strong association between infection with human papillomavirus and oral and oropharyngeal squamous cell carcinoma: a population-based case-control study in southern Sweden. Acta Otolaryngol 2005;125: 1337-1344.

16 Koppikar P, deVilliers EM, Mulherkar R. Identification of human papillomaviruses in tumors of the oral cavity in an Indian community. Int J Cancer 2005;113: 946-950.

17 Lawton G, Thomas S, Schonrock J, et al. Human papillomaviruses in normal oral-mucosa-a comparison of methods for sample collection. J Oral Pathol Med 1992;21:265-269.

18 Campisi G, Panzarella V, Giuliani M, et al. Human papillomavirus: its identikit and controversial role in oral oncogenesis, premalignant and malignant lesions (Review). Int J Oncol 2007;30:813-823.
19 Gillison ML, Koch WM, Capone RB, et al. Evidence for a causal association between human papillomavirus and a subset of head and neck cancers. J Natl Cancer Inst 2000;92:709-720.

20 Kreimer AR, Clifford GM, Boyle P, et al. Human papillomavirus types in head and neck squamous cell carcinomas worldwide: a systematic review. Cancer Epidemiol Biomarkers Prev 2005;14:467-475.

21 Moberg M, Gustavsson I, Gyllensten U. Real-time PCRbased system for simultaneous quantification of human papillomavirus types associated with high risk of cervical cancer. J Clin Microbiol 2003;41: 3221-3228.

22 Dahlgren L, Dahlstrand HM, Lindquist D, et al. Human papillomavirus is more common in base of tongue than in mobile tongue cancer and is a favorable prognostic factor in base of tongue cancer patients. Int J Cancer 2004;112:1015-1019.

23 Clifford GM, Rana RK, Franceschi S, et al. Human papillomavirus genotype distribution in low-grade cervical lesions: comparison by geographic region and with cervical cancer. Cancer Epidemiol Biomarkers Prev 2005;14:1157-1164.

24 Montaldo C, Mastinu A, Quartuccio M, et al. Detection and genotyping of human papillomavirus DNA in samples from healthy Sardinian patients: a preliminary study. J Oral Pathol Med 2007;36:482-487.

25 Campisi G, Giovannelli L, Arico P, et al. HPV DNA in clinically different variants of oral leukoplakia and lichen planus. Oral Surg Oral Med Oral Pathol Oral Radiol Endod 2004;98:705-711.

26 Badaracco G, Rizzo C, Mafera B, et al. Molecular analyses and prognostic relevance of HPV in head and neck tumours. Oncol Rep 2007;17:931-939.

27 De Petrini M, Ritta M, Schena M, et al. Head and neck squamous cell carcinoma: role of the human papillomavirus in tumour progression. New Microbiol 2006; 29:25-33. 